\title{
Design problem solving with biological analogies: A verbal protocol study
}

\author{
HYUNMIN CHEONG, GREGORY M. HALLIHAN, AND L.H. SHU \\ Department of Mechanical and Industrial Engineering, University of Toronto, Toronto, Canada \\ (RECEIVED September 30, 2012; ACCEPTED June 6, 2013)
}

\begin{abstract}
Biomimetic design applies biological analogies to solve design problems and has been known to produce innovative solutions. However, when designers are asked to perform biomimetic design, they often have difficulty recognizing analogies between design problems and biological phenomena. Therefore, this research aims to investigate designer behaviors that either hinder or promote the use of analogies in biomimetic design. A verbal protocol study was conducted on 30 engineering students working in small teams while participating in biomimetic design sessions. A coding scheme was developed to analyze cognitive processes involved in biomimetic design. We observed that teams were less likely to apply overall biological analogies if they tended to recall existing solutions that could be easily associated with specific superficial or functional characteristics of biological phenomena. We also found that the tendency to evaluate ideas, which reflects critical thinking, correlates with the likelihood of identifying overall biological analogies. Insights from this paper may contribute toward developing generalized methods to facilitate biomimetic design.
\end{abstract}

Keywords: Bioinspired Design; Biologically Inspired Design; Biomimetic Design; Conceptual Design; Design Cognition; Protocol Analysis

\section{INTRODUCTION}

In biomimetic or biologically inspired design, designers use analogies found between biological phenomena and design problems to develop design solutions. Although biological analogies have inspired many innovative solutions, many analogies occur by chance and how designers identify and apply those analogies is not fully understood. Therefore, understanding designer behavior during biomimetic design is important for developing systematic methods that support biologically inspired design.

Our interest in analyzing designer behavior in biomimetic design stems from the challenges we observed consistently over a decade (Shu, 2010; Shu et al., 2011). Our approach to supporting biomimetic design is to retrieve potentially useful analogies from biological information readily available in natural-language format. We conducted studies to understand how novice designers identify and transfer analogies from text descriptions of biological phenomena (Mak \& Shu, 2004, 2008; Cheong \& Shu, 2009, 2013a, 2013b; Cheong et al., 2010). In addition, we observed experienced designers

Reprint requests to: L.H. Shu, Department of Mechanical and Industrial Engineering, University of Toronto, 5 King's College Road, Toronto, ON M5S 3G8, Canada. E-mail: shu@mie.utoronto.ca working on application case studies of biomimetic design (Shu et al., 2003; Saitou et al., 2007; Davidson et al., 2009). Both empirical and anecdotal evidence supports that designers have difficulty identifying biological analogies and applying them to develop new solutions. Helms et al. (2009) conducted observational studies on novice designers and reported similar obstacles in biomimetic design.

In biomimetic design, analogical reasoning is an essential cognitive process. To take advantage of strategies found in biology, designers benefit from identifying similarities in underlying principles between biology and engineering. Ideally, designers abstract relationships between functions in order to identify structural similarities ${ }^{1}$ instead of superficial similarities. We believe that analogical reasoning is not a simple associative process but a specific type of associative thinking that requires complex reasoning. Biomimetic design often requires that designers also perform other cognitive processes involved in conceptual design, such as problem formulation, solution generation, analysis, and evaluation.

Our research goal is to gain a better understanding of conceptual design processes that involve biological analogies.

\footnotetext{
${ }^{1}$ In this paper, "structure" means relational structure of a concept, not some physical form.
} 
We therefore chose protocol analysis as the research method and recorded conversations from groups of novice designers working on biomimetic design problems. We identified types of associative thinking involved in biomimetic design and how they influence analogical reasoning. We also analyzed relationships between general conceptual design processes and the use of biological analogies.

The following section provides background in biomimetic design and analogical reasoning. In addition, methods developed for protocol analyses are reviewed to preface our research methods described in Section 3. Section 4 then reports the results, followed by the discussion in Section 5. Section 6 summarizes the main findings and provides remarks on future research.

\section{BACKGROUND}

\subsection{Cognition research in biomimetic design}

Mak and Shu $(2004,2008)$ studied how text descriptions of biological phenomena are used to develop concepts for design problems. The authors observed that biological phenomena that included principles and behaviors in addition to forms tended to be more easily used by students as design stimuli. The authors also found that novice designers tend to fixate on irrelevant features of biological phenomena and incorrectly apply biological strategies to design problems.

Cheong and Shu (2009) observed that biological phenomena containing causally related functions are more likely to serve as useful analogies for design problems. Causal relations often explain how functions are achieved by behaviors. For example, "break down" enables "absorb" in the description "Humans absorb amino acids by breaking down proteins from food." Cheong et al. (2010) and Cheong and Shu (2013a) developed a template to help designers extract strategies from causal relations contained in descriptions of biological phenomena. However, when novice designers used the template in a controlled experiment, analogical transfer only improved marginally.

Helms et al. (2009) and Vattam, Helms, et al. (2010) observed students working on projects in a biologically inspired design course. Helms et al. (2009) reported a number of common errors made by students. Some of these errors may be common to conceptual design in general (e.g., "vaguely defined problems," "poor problem-solution pairing," and "solution fixation"), whereas others are more specific to biomimetic design (e.g., "using off-the-shelf biological solutions," "misapplied analogy," and "improper analogical transfer"). Vattam, Helms, et al. (2010) developed a conceptual framework of compound analogical design that extends existing models of analogy-based design to better represent biologically inspired design. The authors also identified the distribution of analogies across different conceptual design phases.

In summary, complex cognitive processes are involved in biomimetic design, and designers often exhibit maladaptive behaviors when applying biological analogies in conceptual design.

\subsection{Other research in biomimetic design}

Other previous research in biomimetic design has focused on developing models to support the cataloging and use of biological information. A number of studies demonstrated that representation modes have significant influence on design by analogy (Linsey et al., 2008; Sarkar \& Chakrabarti, 2008; Helms et al., 2010; Jin \& Benami, 2010).

Goel et al. (2011) represent causal processes between states using the structure-behavior-function (SBF) framework and observed that the SBF model of biological systems helps novice designers understand complex relations, such as causality, in systems. They also note that DANE, a library of SBF models of biological systems, could potentially be used as a concept generation tool.

Sartori et al. (2010) used SAPPhIRE constructs to represent mechanisms of transfer in 20 biomimetic examples in the literature. SAPPhIRE, developed by Chakrabarti et al. (2005), defines multiple levels of abstraction in order to explain how a biological system works to fulfill its goals. The authors found that successful biomimetic examples usually involve systems that share similarities at higher levels of abstraction.

Nagel, Nagel, et al. (2010) developed a framework that is primarily based on functional modeling of biological systems with a set of terms from the "engineering-to-biology thesaurus" (Nagel, Stone, et al., 2010). Although the authors provide a detailed description for using their technique, they have not empirically studied its direct benefits to designers or how designers use it in practice.

The above biomimetic design models are effective at formally representing complex biological systems in consistent format. However, their utility in concept generation may require further validation. For instance, Vattam, Wiltgen, et al. (2010) reported the challenges of using SBF modeling in concept generation. They noted that novice designers were not willing to build models without seeing the direct benefits and were not convinced of DANE's usefulness and value.

We propose that better understanding of cognition involved in biomimetic design will help improve these models and ultimately lead to more effective biomimetic concept generation. In the following section, we discuss background research in analogical reasoning, which is fundamental to biomimetic concept generation.

\subsection{Background in analogical reasoning}

Analogical reasoning is considered to be central to creative thought (Holyoak \& Thagard, 1995; Goel, 1997; Ward, 1998; Gentner, 2003). In design, analogical reasoning allows individuals to find similarities between a source knowledge space and a target design space, and transform the source knowledge into new design solutions.

Gentner (1983) identifies two levels at which similarities can be found in analogical reasoning: superficial and relational. The superficial level refers to object attributes. The 
relational level can be further decomposed into two levels: relations between objects and relations between relations, or "higher order relations."

In the context of biomimetic design, we assert that the superficial level corresponds to the attributes of biological entities (objects). The relation between objects would then correspond to the functions of biological entities, and the relation between relations would correspond to the strategy enabled by the functions of biological entities. We describe the different characteristic levels of enzymes in the following examples:

- entity: enzymes are ribbon shaped

- function: enzymes bind to substrates or enzymes perform biochemical activities

- strategy: enzymes bind to substrates to perform biochemical activities

Several researchers (Gentner, 1983; Holyoak \& Thagard, 1989; Markman \& Gentner, 1993) agree that relational structure should be matched between two concepts during analogical reasoning. In particular, a higher order relation (e.g., a causal relation) provides "systematicity" in which a set of relations can be aligned and transferred (Gentner, 1983; Markman \& Gentner, 1993).

Goel (1997) states, "[A]nalogical transfer requires the use of generic abstractions, where the abstractions typically express the structure of relationships between generic types of objects and processes." In biomimetic design, designers must abstract information in biological phenomena to identify relational similarities to design solutions.

\subsection{Protocol analysis for design research}

Protocol analysis is an effective research method to study the cognition of designers. Cross et al. (1996) state that protocol analysis can offer the benefits of both observational and experimental research. Qualitative observations on verbalized thoughts can reveal interesting behaviors in design. Protocols can also be coded to produce experimental data and enable rigorous analysis.

Concurrent verbalization, or "think-aloud" protocol (van Someren et al., 1994), is often used to elicit verbalized thoughts from designers. Verbalized thoughts are presumed to reflect the designers' underlying cognition. Ericsson and Simon (1993) concluded that concurrent verbalization does not affect the thought process. However, with tasks that involve complex cognitive processes such as design, concurrent verbalization could be perceived as additional cognitive workload. Chiu and Shu (2010) report some limitations of the think-aloud protocol.

Design researchers often collect protocols from groups of designers participating in design processes and talking aloud to one another as in natural settings (Cross et al., 1996). While this approach may not capture cognitive mechanisms in as much detail, the process is more natural and may better reflect actual design practices. Another method is to conduct retro- spective protocol analysis (van Someren et al., 1994), where after design sessions, designers talk about their thought processes that occurred during the design sessions.

\subsubsection{Analyzing design activities in verbal protocols}

A number of analytical methods have been developed for protocol analysis. Merriam (2009) recommends that the analysis of qualitative data, such as design protocols, should ultimately be tailored toward the needs of the researcher. In general, researchers develop coding schemes to identify categories of interest. Miles and Huberman (1994) suggest that these categories should be meaningful and clearly defined.

Goldschmidt (1995) used design "moves," which identify ideas that transform the design situation and reflect the development of ideas. Kvan and Gao (2006) adopted Schön's (1983) definition of design processes: "framing," "moving," and "reflecting," to study the problem-framing process in design. Gero (2010a) proposed a coding scheme based on the function-behavior-structure ontology (Gero, 1990) to serve as a generalized framework for protocol analysis. The coding scheme has been used in a number of studies (Gero \& McNeill, 1998; Kan et al., 2007; Bilda \& Gero, 2008; Gero, 2010b). Recently, Dinar et al. (2012) proposed the problem map model, which is another domain-independent coding scheme but intended to focus on the analysis of problem formulation in design.

Another widely used method for protocol analysis is linkography, developed by Goldschmidt (1990). Researchers construct linkographs by linking coded design segments that are related. Linkography has been used to examine a wide variety of phenomena in design, including problem framing effects (Kvan \& Gao, 2006), visuospatial working memory load (Bilda \& Gero, 2008), and design fixation (Gero, 2010b). The analysis of linkographs has also progressed to include applying information processing models such as cluster analysis (Bilda \& Gero, 2008) and entropy models (Kan et al., 2007). These techniques help researchers quantify the relationships found in linkographs.

Computational linguistic techniques are also used to analyze design protocols. Dong $(2005,2006,2007)$ used latent semantic analysis to quantify coherent thinking and lexical chain analysis to evaluate concept formation in design teams. These techniques provide more objective and standardized ways to analyze protocols.

\section{METHODS}

This section reports the details of our experiment, data collection, and coding scheme for protocol analysis.

\subsection{Participants}

Thirty-four engineering students ( 32 males, 2 females) participated in a biomimetic design laboratory session in a mechanical design course at the University of Toronto. All students were in the final year of their undergraduate programs in ei- 
ther mechanical engineering or the manufacturing stream of an engineering honors program. Most students had taken previous courses that involve designing in teams, in addition to a first-year project-based design course required for every engineering student at the University of Toronto. Prior to the laboratory session, the students received $2 \mathrm{~h}$ of lecture on examples and research challenges in biomimetic design; however, none of the problems or biological phenomena used for the experiment were discussed. After reviewing the types of correspondence that can occur in biomimetic design described by Mak and Shu (2004), the experiment was conducted during the second part of the tutorial/laboratory session. Thirty of the 34 students consented to have their design sessions audiorecorded for research purposes. Four students were uncomfortable being audiorecorded but were happy to share their experiences for both teaching and research purposes. Therefore, the quantitative analyses were based on the transcripts of the 30 students who were audiotaped, but notable insights that came from the 4 students who were not audiotaped are described qualitatively in the Discussion.

\subsection{Experimental design}

The design exercise required students to generate solutions for a problem using biological analogies. Three design problems were used, and each problem was paired with a description of a biological phenomenon as the source of analogy. The goal of using multiple problems was to observe participants perform analogical reasoning in different contexts, although it comes at the cost of decreasing experimental data for any given problem. The students were explicitly asked to use the given biological phenomena as analogies. How- ever, they were neither encouraged nor restricted from making analogies to other biological phenomena from their knowledge. Furthermore, they did not access materials other than those provided.

Three to four students were randomly assigned to a team and each team worked on a single design problem. This experimental design produced verbal protocols from 9 different teams, which we believe provide a significant amount of data from which one can draw insights on the analogical reasoning process. The number of teams in past verbal protocol studies vary from 6 (Kan et al., 2007; Bilda \& Gero, 2008) to 18, which were further divided into three experimental groups of six (Kvan \& Gao, 2006).

A combination of time, space, and staffing limitations led to one unrecorded and three recorded laboratory stations in the same room where problem solving occurred simultaneously. However, the recorded stations were at least $30 \mathrm{ft}$ away from each other, and conversations were not comprehensible between stations. The unrecorded station was smaller, with many fewer participants who did not need to speak as loudly, and could therefore be located closer to, without increased influence on, the recorded stations. Figure 1 shows the physical environment of the experiment.

Each recorded team was assigned to one of the three main stations. Table 1 shows this assignment and the order in which each team solved one of the three design problems at each station. The order of problems was counterbalanced in a $3 \times 3$ Latin square matrix to control for order effect and station effect. While one team was solving a design problem, the other two teams in the same station were instructed to observe the active team. Specifically, the observing teams were instructed to determine and evaluate the types of similarities

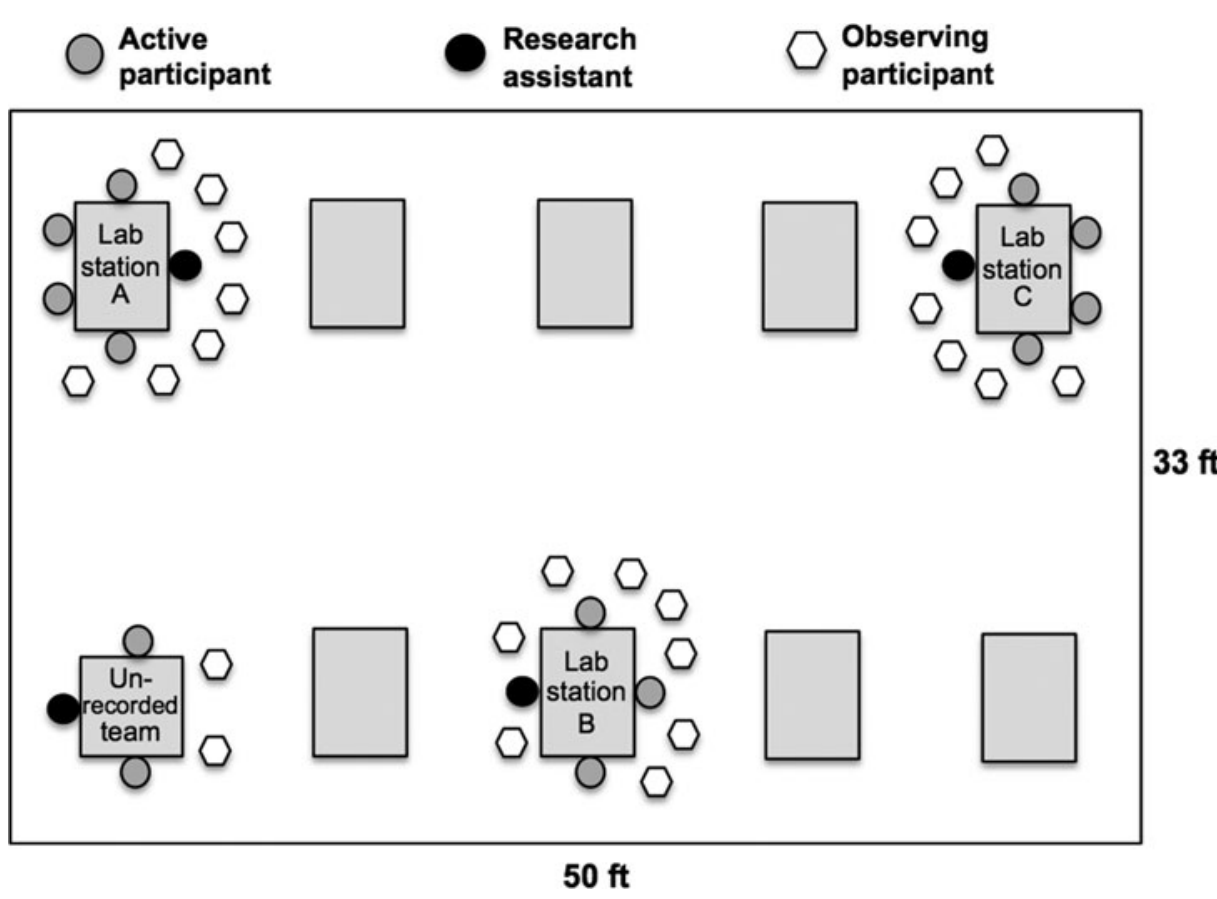

Fig. 1. The physical environment of the experiment. 
Table 1. Details on design teams and problems assigned

\begin{tabular}{cccl}
\hline \hline $\begin{array}{c}\text { Lab } \\
\text { Station }\end{array}$ & $\begin{array}{c}\text { Design } \\
\text { Team No. }\end{array}$ & No. of Students & \multicolumn{1}{c}{ Design Problem } \\
\hline A & 1 & 4 & Promotional mailing \\
& 2 & 3 & Authorized disassembly \\
& 3 & 3 & Wet scrubber \\
B & 4 & 3 & Wet scrubber \\
& 5 & 4 & Promotional mailing \\
& 6 & 3 & Authorized disassembly \\
C & 7 & 3 & Wet scrubber \\
& 8 & 4 & Promotional mailing \\
& 9 & 3 & \\
\hline \hline
\end{tabular}

made by the active team and note when and how often the similarities occurred. While we were hoping to use the peer evaluations of the students as additional data, we found that they were not sufficiently consistent for research purposes and therefore do not discuss them further. It is reasonable to expect the presence of a learning effect for the second and third active team at each station. However, because a different problem was solved at each station at each round, the learning effect would be consistent across problem groups. Furthermore, multiple reviews of recorded protocols during the analysis did not reveal any significant effect owing to learning.

For the four students who were not audiorecorded, the three problems were divided among different pairings that formed two-person active teams. This group then followed the same procedure as the recorded groups with these smaller active teams. A research assistant assigned to this group also followed the same procedure as for the audiorecorded groups, but observations of this group were recorded using written notes.

At the beginning of each design session, each member of the active team was provided with a written copy of the design problem and corresponding biological phenomenon. While each team was given $20 \mathrm{~min}$ to generate solutions for the design problem owing to time constraints of the scheduled laboratory period, our past experiments have suggested that 20 min are sufficient to evaluate analogical reasoning from given biological phenomena. One team (Group 9) in the current study used only $12 \mathrm{~min}$ and stated that they could not generate any more solutions.

\subsection{Design problems and biological phenomena}

The design teams received the following descriptions of design problems and corresponding biological phenomena selected by the researchers. The first problem and biological phenomenon was adopted from Saitou et al. (2007). The second and third problems were devised by first identifying interesting biological phenomena and then creating problems that could be solved based on the biological phenomena. Only text descriptions of biological phenomena were given.

1. a. Authorized disassembly problem: Original equipment manufacturers (OEMs) want easy disassembly of their products to reduce disassembly cost and increase the net profit from reuse and recycling at product end of life. However, OEMs are also concerned with protecting high-value components from theft and access by competitors. How can you allow disassembly that is easy but only by those authorized?

b. Biological phenomenon (enzyme-substrate interaction): Enzymes are complex proteins that bind to specific substrates (molecules) and form enzymesubstrate complexes that perform biochemical activities. The specific binding is achieved when the active site of an enzyme geometrically matches its corresponding substrate. However, an enzyme changes its shape with environmental factors such as $\mathrm{pH}$ and temperature. This shape change alters the conformation of the enzyme's active site to the point where substrates can no longer fit, thereby disabling the function of the enzyme-substrate complex.

2. a. Promotional mailing problem: You are a marketing director for a credit card company. You are looking for an effective strategy to distribute sign-up promotional mailings within a city. You would like to distribute promotional mail to selected neighborhoods in the city so that a large proportion of the promotional mail actually results in people signing up. In other words, you do not want to waste resources on sending promotional mail to neighborhoods where people are not likely to sign up. Assuming that you do not have any demographic information of the city, how would you optimize the use of promotional mailings?

b. Biological phenomenon (ant foraging): An ant colony can identify the shortest path between its nest and food source with the following strategy. Ants depart the colony to search randomly for food, laying down pheromones on the trail as they go. When an ant finds food, it follows its pheromone trail back to the nest, laying down another pheromone trail on the way. Pheromones have more time to dissipate on longer paths and less time to dissipate on shorter paths. Shorter paths are also traveled more often relative to longer paths, so pheromones are laid down more frequently on shorter paths. Additional ants follow the strongest pheromone trails between the food source and the nest, further reinforcing the pheromone strength of the shortest path.

3. a. Wet scrubber problem: Wet scrubbers are air pollution control devices that remove pollutants from industrial exhaust systems. In conventional wet scrubbers, exhaust gas is brought into contact with a liquid solution that removes pollutants from the gas by dissolving or absorbing them into the liquid. The removal efficiency of pollutants is often improved by increasing the contact time or the contact area be- 
tween the exhaust gas and the scrubber liquid solution. What other strategy could be used to increase the removal efficiency of wet scrubbers?

b. Biological phenomenon (penguin's circulatory system): Penguins are warm-blooded yet keep their uninsulated feet at a temperature close to freezing to minimize heat transfer to the environment. The veins that carry cold blood from the feet back to the body are located close to the arteries that carry warm blood from the body to the feet. The warm blood flows in the opposite direction as the cold blood, which allows the penguins to transfer the most heat to the cold blood. This reduces both the amount the returning blood can drop the core body temperature and the amount of heat lost through the feet.

\subsection{Recording design protocols}

Students in each design team were instructed to verbalize their ideas during the design process; these verbalizations were audiorecorded and transcribed for analysis. A research assistant was assigned to each laboratory station to facilitate and audiorecord the design sessions. To control for any confounding effects introduced by the research assistants, they were provided with a script to handle potential questions from students and instructed not to contribute to the design process. The research assistants only interceded when design progress slowed or the students had settled on a design solution. After $20 \mathrm{~min}$, the research assistants gave the next active team its corresponding design problem.

Two authors of this paper transcribed the audio files for each design team. After each transcript was generated, it was cross-reviewed by the other researcher to verify its accuracy. Some audio data were not interpretable (e.g., multiple designers speaking at once, or speaking too quietly or unintelligibly), and such data were excluded from further analysis.

In addition to verbalization, participants were also allowed to write down or sketch ideas on paper to share them among team members during the design process. These ideas were also collected by research assistants and later used to support the analysis of verbal protocols.

\subsection{Protocol coding}

We developed our own coding scheme because existing coding schemes could not capture the different types of associative thinking processes of interest to us. In addition, we wanted to be able to identify cognitive processes that may be unique to biomimetic design. Our method of coding design protocols into a set of defined categories is in line with other protocol analyses identified in Section 2.4.1.

Segmenting the protocols by participant ideas or turns was difficult due to multiple interruptions from other team members and many instances of incomplete ideas. Instead, we chose to segment each protocol into 10-s units to provide cod- ers with manageable chunks of protocols to evaluate. However, for both coding schemes detailed below, the segment length is inconsequential because we counted all the similarity comparisons that occurred during each time segment, and if a segment contained multiple design activity modes, we divided them accordingly.

After segmenting the protocols, the coders determined whether each time segment contained events of interest described in the following two subsections.

\subsubsection{Identifying the levels of similarity comparisons}

We first coded participants' utterances that involved similarity comparisons into three categories that are consistent with Gentner's (1983) theoretical framework for analogical reasoning (outlined in Section 2.3). These categories are the following:

- entity: a comparison to superficial characteristics of entities of the biological phenomenon

- function: a comparison to functions of the biological phenomenon

- strategy: a comparison involving a higher order relation (strategy) from the biological phenomenon

Multiple types of comparisons were possible in a single segment, because the strategy-level comparison often invokes comparisons at the entity and functional levels. The coders therefore identified multiple levels of comparisons for each segment as they occurred. If a particular similarity comparison occurred over two or more segments, all the segments containing the similarity comparison are coded with the relevant category.

Figure 2 shows a sequence of an example protocol coded for the above similarity comparison categories. The time elapsed is shown on the top of the figure. The shaded numbers correspond to the number of comparisons of the type labeled to the left that occurred in that 10-s interval. For example, during both 10-s time segments between 260 and $280 \mathrm{~s}$ into the design task, one comparison at the entity level was coded. This coding could represent either a single entity-level comparison that spanned two time segments or a different entity-level comparison that occurred in each segment. In contrast, during both 10-s segments between 440 and $460 \mathrm{~s}$ into the design task, two entity-level comparisons were coded. Such coding usually corresponds to two different entity-level comparisons in each of the two time segments. In a few instances, such coding corresponds to one comparison that spanned two segments and two separate comparisons in each of the segments. Table 2 shows examples of similarity comparisons identified for each coding category for the wet scrubber problem.

\subsubsection{Identifying design activity modes}

In addition to the levels of similarity comparisons, the coders identified different design activity modes. Five different categories were used: 


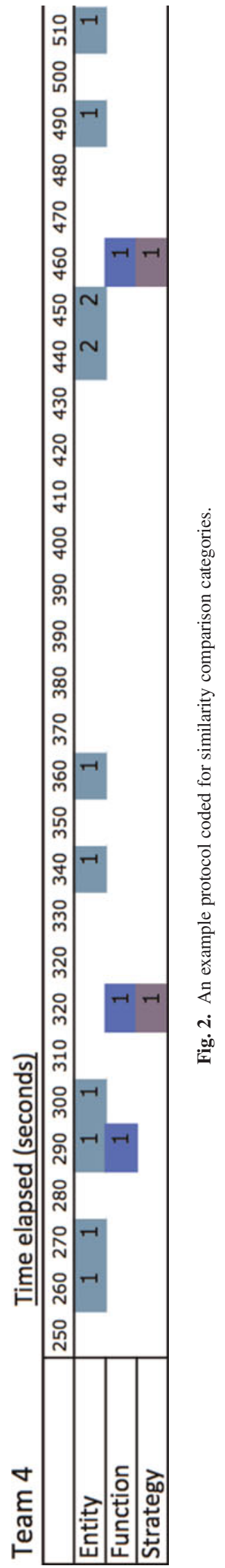

Table 2. Examples of similarity comparisons identified for the wet scrubber problem

\begin{tabular}{|c|c|c|}
\hline $\begin{array}{l}\text { Similarity } \\
\text { Comparison }\end{array}$ & Coding & Example Passage $^{a}$ \\
\hline Entity & E & $\begin{array}{l}\text { "Veins have a lot of surface area so we can make } \\
\text { sure that ... I mean ... the liquid we are using } \\
\text { for the scrubbing, it can go through like really } \\
\text { narrow pipes or whatever to increase the surface } \\
\text { area." ... }\end{array}$ \\
\hline Function & $\mathrm{F}$ & $\begin{array}{l}\text { "We also did kind of blood circulation, "cause uh, } \\
\text { we are recirculating [scrubber solution and } \\
\text { exhaust gas]." }\end{array}$ \\
\hline \multirow[t]{2}{*}{ Strategy } & S & $\begin{array}{l}\text { "It says the opposite direction allows, like, most } \\
\text { flow of gas exchange ... so make, I don't know, } \\
\text { maybe we could make the . . }\end{array}$ \\
\hline & S & $\begin{array}{l}\text { liquid scrubber run in one direction, and ... gas run } \\
\text { in the other direction. That increases the flow } \\
\text { [exchange]." }\end{array}$ \\
\hline
\end{tabular}

${ }^{a}$ Extracted from one or two consecutive 10-s segments.

- problem analysis: participants discuss or analyze the problem

- biological phenomenon: participants discuss or analyze the biological phenomenon

- existing solution: participants relate to or recall existing solutions

- new solution: participants generate new solutions

- evaluation: participants evaluate their solutions or analogies formed

The categories include both essential activities of general conceptual design and important activities unique to biomimetic design. For example, in addition to the problem analysis common to all conceptual design, our participants also discussed the biological phenomenon presented as stimuli. Furthermore, we distinguished between two categories for solution generation: recalling existing solutions from prior knowledge and developing new solutions. This distinction is informed by previous studies reporting that designers often use biological analogies to recall existing solutions rather than develop new solutions (Mak \& Shu, 2008; Helms et al., 2009). We coded a segment as recalling an existing solution only if participants explicitly named or made reference to an existing solution, for example, "use telemarketing surveys" or ". . like Apple making its own interfaces." Evaluation refers to critiquing either developed solutions or analogies formed. Table 3 shows examples of each design activity mode identified from the protocols for the authorized assembly problem.

The five categories are mutually exclusive. When a segment contained more than one design activity mode, the segment was divided into the corresponding number of modes identified. Figure 3 shows coding for design activity modes for the same segment of protocol shown in Figure 2. This portion does not include an instance where a segment was divided to accommodate multiple activity modes. 
Table 3. Examples of design activity modes for the authorized disassembly problem

\begin{tabular}{|c|c|c|}
\hline $\begin{array}{c}\text { Design Activity } \\
\text { Mode }\end{array}$ & Coding & Example Passage ${ }^{a}$ \\
\hline Problem analysis & $\mathrm{P}$ & $\begin{array}{l}\text { "At the same time, they don't want it to be so } \\
\text { easy . . . umm . . . people can't just } \\
\text { disassemble it themselves and take it out." }\end{array}$ \\
\hline $\begin{array}{l}\text { Biological } \\
\text { phenomenon }\end{array}$ & B & $\begin{array}{l}\text { "So that means if enzymes are faced under } \\
\text { different, umm, environment, then the } \\
\text { binding, kind of, disintegrates. They can't } \\
\text { bind together anymore because they don't } \\
\text { fit." }\end{array}$ \\
\hline Existing solution & Ex & $\begin{array}{l}\text { "It's like Apple making the laptop last time. } \\
\text {. the memory was attached to the laptop } \\
\text { itself, the motherboard, so it's hard to } \\
\text { disassemble ..." }\end{array}$ \\
\hline New solution & $\mathrm{S}$ & $\begin{array}{l}\text { "You could have fasteners that change } \\
\text { shape, depending on ... maybe something } \\
\text { like, running electric current." }\end{array}$ \\
\hline Evaluation & Ev & $\begin{array}{l}\text { "Um, so that would work pretty nicely but it } \\
\text { also may increase the, the cost, of } \\
\text { disassembly if we were using a special } \\
\text { part like that." }\end{array}$ \\
\hline
\end{tabular}

${ }^{a}$ Segments identified to contain a specific design activity mode.

\subsubsection{Coding reliability}

Two of the authors first coded the protocols to identify similarity comparisons. The authors were deemed to be appropriate as coders because coding similarity comparisons requires theoretical knowledge in analogical reasoning.

Assessing interrater reliability for the similarity comparison coding was challenging. Because the similarity comparison categories were not mutually exclusive and not all protocol segments contained these coded events, the standard methods of calculating interrater reliability could not be applied. For example, each segment could contain any number of entity- and function-level comparisons, so the possible number of outcomes for each observation was theoretically infinite. Therefore, we decided that producing final coding results based on the consensus of multiple coders was more important and adapted the Delphi method (Linstone \& Turoff, 1975). Gero and McNeill (1998) provide detailed descriptions of how the Delphi method could be used for verbal protocol studies. For the current research, the coders first individually coded one third of the protocols, after which cases of disagreement were arbitrated until agreement was reached. The coders then individually completed the rest of the protocols, followed by another arbitration process to achieve a single set of coding results. We did, however, calculate the percentage agreement on protocol segments that contained the strategy-level comparison, because each segment never contained more than one strategy-level comparison and the possible outcome for this assessment was binary. The raters showed $92 \%$ agreement on the strategy-level comparison before the final arbitration process.

Coding design activity modes involved identifying one of mutually exclusive activity modes for each segment. There-

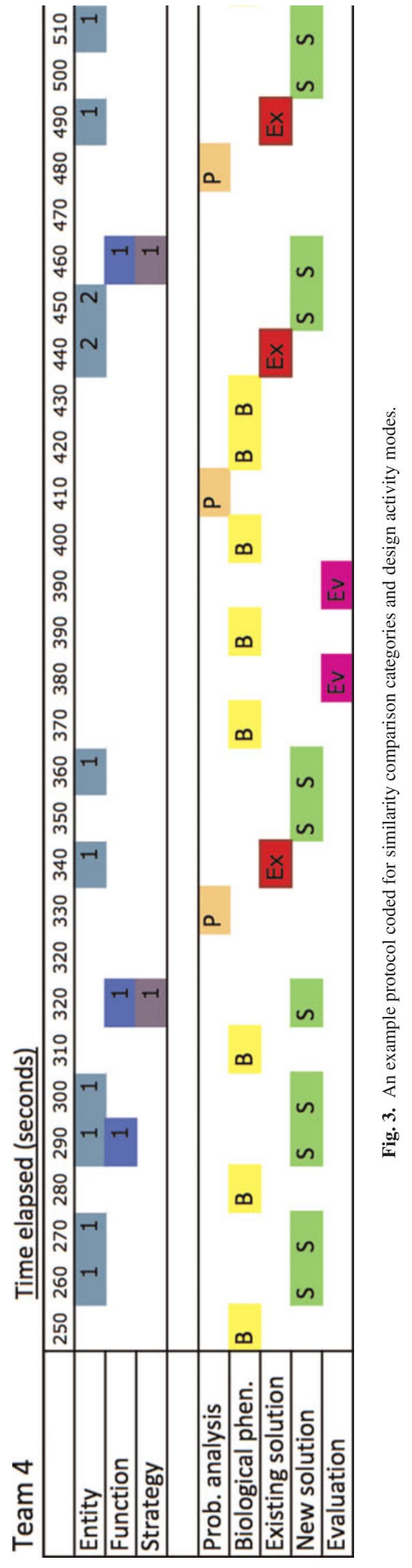


Table 4. Frequency of coded events and associated measures for each design team

\begin{tabular}{|c|c|c|c|c|c|c|c|c|c|}
\hline & \multicolumn{3}{|c|}{ Authorized Disassembly } & \multicolumn{3}{|c|}{ Promotional Mailing } & \multicolumn{3}{|c|}{ Wet Scrubber } \\
\hline & Team 2 & Team 6 & Team 7 & Team 1 & Team 5 & Team 9 & Team 3 & Team 4 & Team 8 \\
\hline Total active time segments & 104 & 64 & 121 & 84 & 82 & 71 & 85 & 106 & 97 \\
\hline \multicolumn{10}{|l|}{ Similarity comparison } \\
\hline Entity & $\begin{array}{c}23.1 \% \\
(24)\end{array}$ & $\begin{array}{c}18.8 \% \\
(12)\end{array}$ & $\begin{array}{c}21.5 \% \\
(26)\end{array}$ & $\begin{array}{l}6.0 \% \\
(5)\end{array}$ & $\begin{array}{c}20.7 \% \\
(17)\end{array}$ & $\begin{array}{c}24.6 \% \\
(17)\end{array}$ & $\begin{array}{l}9.2 \% \\
(8)\end{array}$ & $\begin{array}{c}27.4 \% \\
(29)\end{array}$ & $\begin{array}{c}12.4 \% \\
(12)\end{array}$ \\
\hline Function & $28.8 \%$ & $7.8 \%$ & $19.0 \%$ & $7.1 \%$ & $14.6 \%$ & $23.0 \%$ & $10.8 \%$ & $3.8 \%$ & $21.6 \%$ \\
\hline Strategy & $22.1 \%$ & $0.0 \%$ & $2.5 \%$ & $25.0 \%$ & $17.1 \%$ & $8.2 \%$ & $1.5 \%$ & $1.9 \%$ & $9.3 \%$ \\
\hline \multicolumn{10}{|l|}{ Design activity mode } \\
\hline Problem analysis & $14.4 \%$ & $17.2 \%$ & $23.1 \%$ & $17.9 \%$ & $9.8 \%$ & $9.8 \%$ & $7.7 \%$ & $17.0 \%$ & $9.3 \%$ \\
\hline Biological phenomenon & $8.7 \%$ & $17.2 \%$ & $16.5 \%$ & $16.7 \%$ & $19.5 \%$ & $27.9 \%$ & $13.8 \%$ & $33.0 \%$ & $13.4 \%$ \\
\hline Existing solution & $5.8 \%$ & $18.8 \%$ & $19.8 \%$ & $4.8 \%$ & $8.5 \%$ & $8.2 \%$ & $10.8 \%$ & $14.2 \%$ & $7.2 \%$ \\
\hline New solution & $55.8 \%$ & $42.2 \%$ & $31.4 \%$ & $45.2 \%$ & $54.9 \%$ & $44.3 \%$ & $60.0 \%$ & $32.1 \%$ & $55.7 \%$ \\
\hline Evaluation & $15.4 \%$ & $4.7 \%$ & $9.1 \%$ & $15.5 \%$ & $7.3 \%$ & $9.8 \%$ & $7.7 \%$ & $3.8 \%$ & $14.4 \%$ \\
\hline New solution generation segments & $323 \%$ & $00 \%$ & $76 \%$ & $387 \%$ & $226 \%$ & $166 \%$ & $21 \%$ & $54 \%$ & $146 \%$ \\
\hline
\end{tabular}

Note: The percentages (rounded to one decimal place) refer to the count of each coded event per the total number of active time segments. For the Entity category, the raw count of its occurrence is shown as an example.

fore, the Cohen $\kappa$ was used to calculate interrater reliability between the lead author and another independent coder for $30 \%$ of the transcript. The second coder was a PhD student conducting research in design theory and methodology. This coder did not receive any training but did receive the examples shown in Table 3 as a guideline. The two coders agreed on $85 \%$ of the codes with $\kappa=0.79$, corresponding to agreement that is "substantial" according to Landis and Koch (1977) and "excellent" according to Fleiss (1981). Given this agreement, we based our analysis on the lead author's complete set of coding results.

\section{RESULTS}

Presented first are problem group comparisons and correlation analyses based on the coded events reported in Table 4 . The frequency of each coded event is determined by the percentage of active time segments dedicated to that event. Time segments are considered active if one of the coded design activity modes occurred. However, not all active time segments involved similarity comparisons. The last measure (percentage of new solution generation segments that involve strategy-level comparison) indicates each team's tendency to develop new analogous solutions. We begin by comparing teams within each problem group to identify tendencies of individual teams that either detracted from or contributed to developing solutions that are analogous to the biological phenomenon provided.

\subsection{Authorized disassembly problem}

Figure 4 shows differences in the frequency of similarity comparisons made between the three teams that solved the

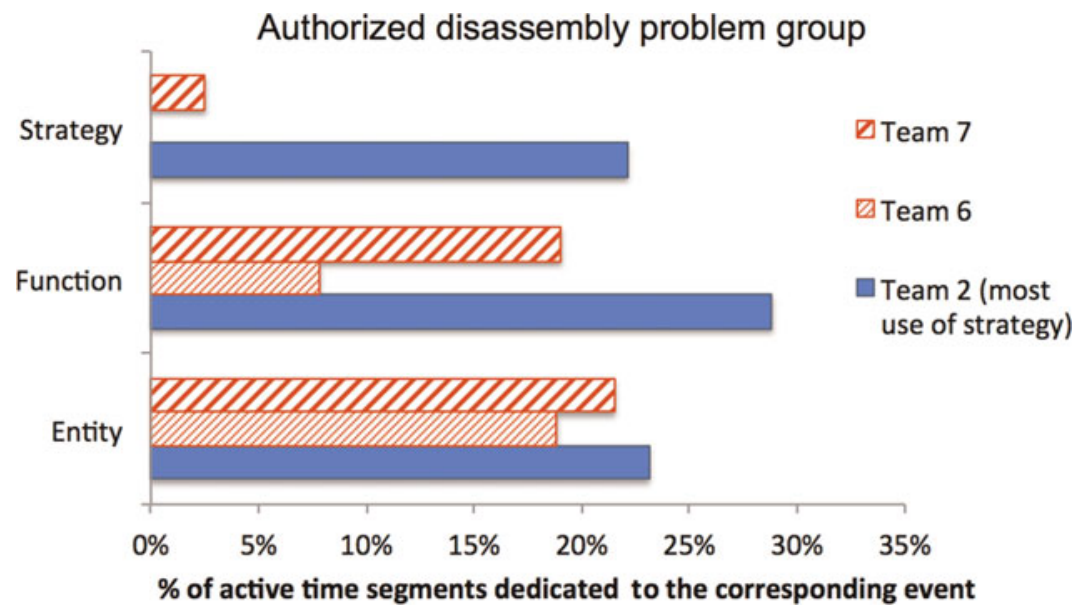

Fig. 4. The frequency of similarity comparisons by the three teams in authorized disassembly problem group. 


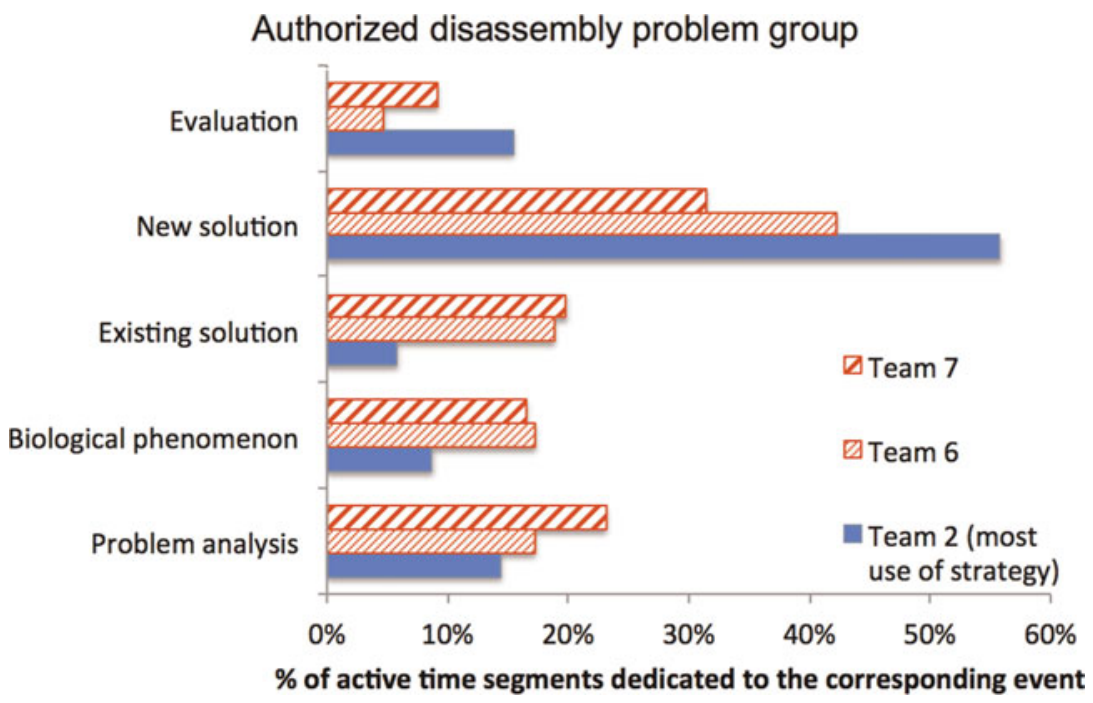

Fig. 5. Frequency of design activity modes by the three teams in authorized disassembly problem group.

authorized disassembly problem. Only Team 2 was able to develop solutions based on the expected analogy, with $22.1 \%$ of the active design segments involving strategylevel comparisons. In contrast, Team 6 could not develop any solutions based on the analogy, and only $2.5 \%$ of Team 7's active design segments involved strategy-level comparisons.

Team 2 was the only team of the three that made more function-level comparisons than entity-level comparisons. In contrast, Team 6, who did not develop any solution based on the expected analogy, made twice as many entity-level comparisons as function-level comparisons.

Figure 5 shows the frequency of design activity modes used by each team. Team 2 spent more time developing new solutions and evaluating their solutions, and less time recalling existing solutions and discussing the biological phenomenon than did the other teams.

\subsection{Promotional mailing problem}

All three teams that worked on the promotional mailing problem used the underlying strategy from the ant foraging phenomenon to generate solutions. Interestingly, the frequency of strategy-level comparisons was inversely proportional to the frequency of both entity-level and function-level comparisons. Figure 6 shows the frequency of similarity comparisons made by each team. Team 1, with $25.0 \%$ strategy-level comparisons, spent $6.0 \%$ of their active time making entity-level comparisons, whereas Team 9, with $8.2 \%$ strategy-level comparisons, spent $24.6 \%$ of their active time making entity-level comparisons.

Figure 7 shows the frequency of design activity modes used by each team. Less dramatic differences in the frequency of design activity modes were observed between Team 1 and Team 9, the teams that made the most and the least use of

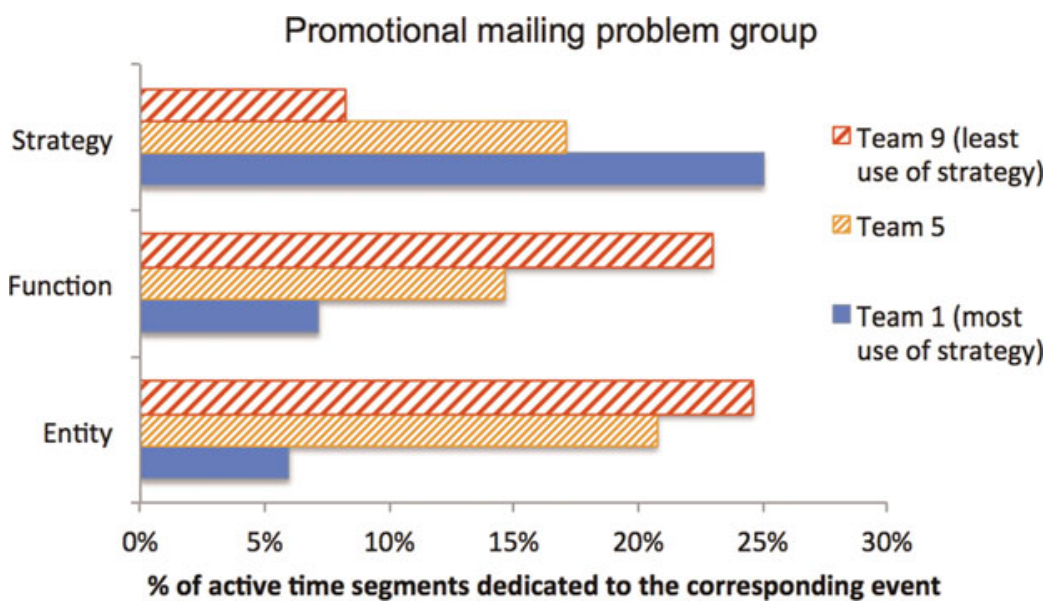

Fig. 6. Frequency of similarity comparisons by the three teams in promotional mailing problem group. 


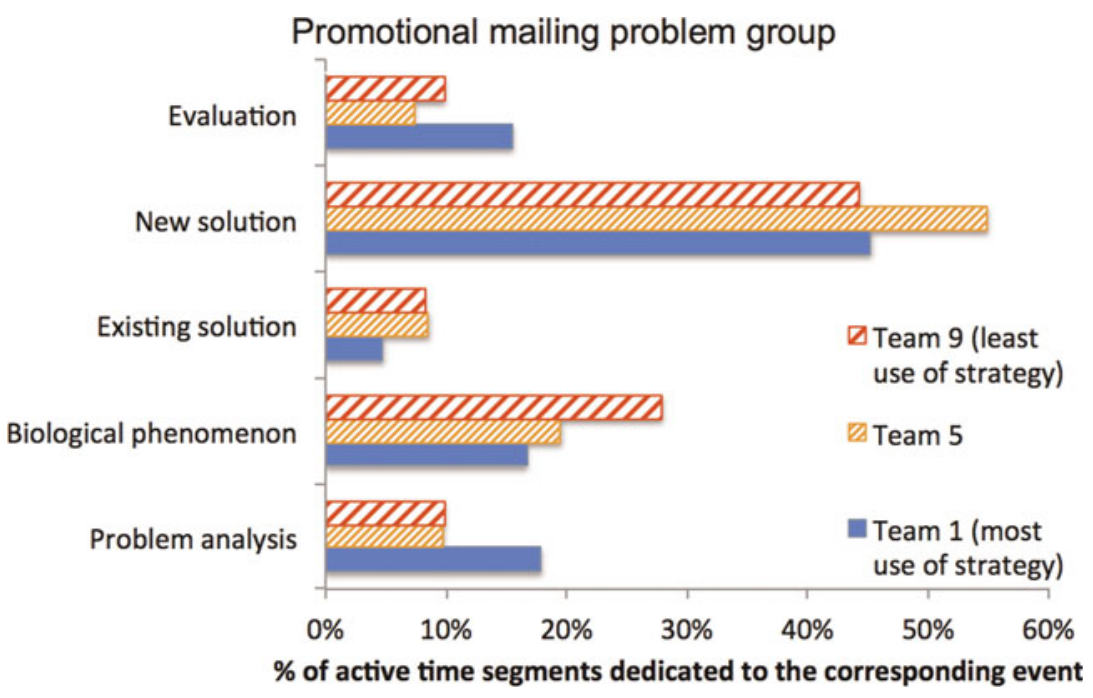

Fig. 7. Frequency of design activity modes by the three teams in promotional mailing problem group.

strategies, respectively. Team 1 spent more time on evaluation and problem analysis, while Team 9 spent more time recalling existing solutions and discussing the biological phenomenon.

\subsection{Wet scrubber problem}

Figure 8 shows the frequency of similarity comparisons made by the teams that solved the wet scrubber problem. Team 8 had $9.3 \%$ of its active design segments involving strategylevel comparisons, while the other two teams had $1.5 \%$ and $1.9 \%$ for the same measure. Similar to the authorized disassembly problem group, the team that spent the most time developing analogous solutions also had the highest ratio of function-level comparisons to entity-level comparisons. Team 4 had more than twice as many entity-level comparisons as the other two teams.

Figure 9 shows the frequency of design activity modes used by each team. Similar to the other two problem groups, the team (Team 8 ) that spent the most time developing anal- ogous solutions also spent the most time on evaluation and the least time recalling existing solutions. In addition, the team (Team 4) that spent the least time developing new solutions spent the most time discussing the biological phenomenon and analyzing the problem.

\subsection{Comparing design problem groups}

We noticed that all three teams who worked on the promotional mailing problem used the underlying strategy from the ant foraging phenomenon to generate solutions. However, the majority (two thirds) of the teams that worked on the authorized disassembly problem or the wet scrubber problem could not develop or rarely developed solutions based on the underlying strategy.

Figure 10 compares the frequency of similarity comparisons between the problem groups, but none of the differences between groups were statistically significant. Figure $11 \mathrm{com}-$ pares the frequency of design activity modes performed by

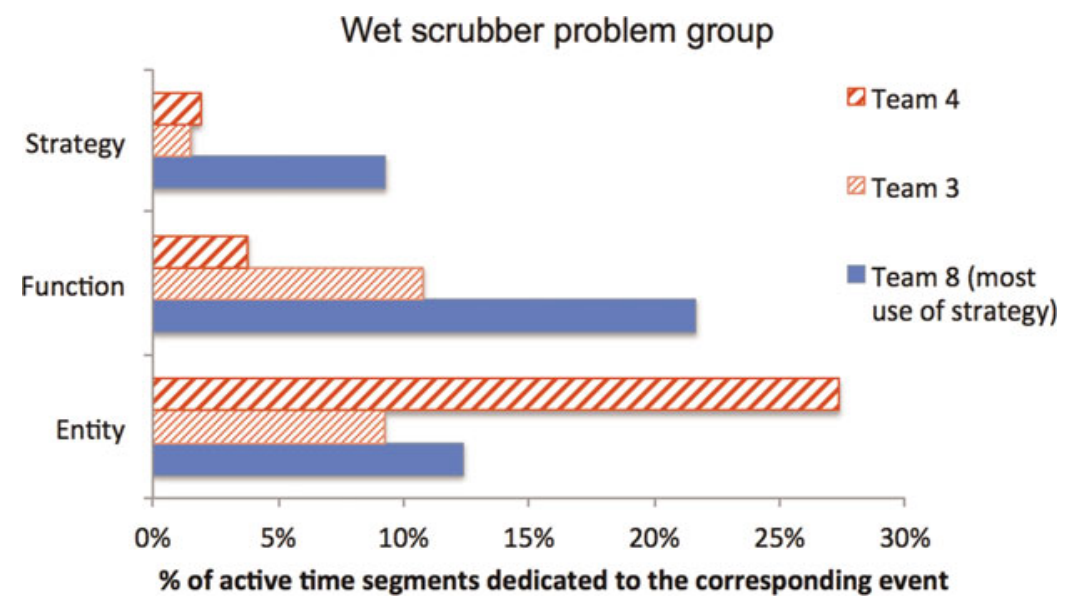

Fig. 8. Frequency of similarity comparisons by the three teams in wet scrubber problem group. 


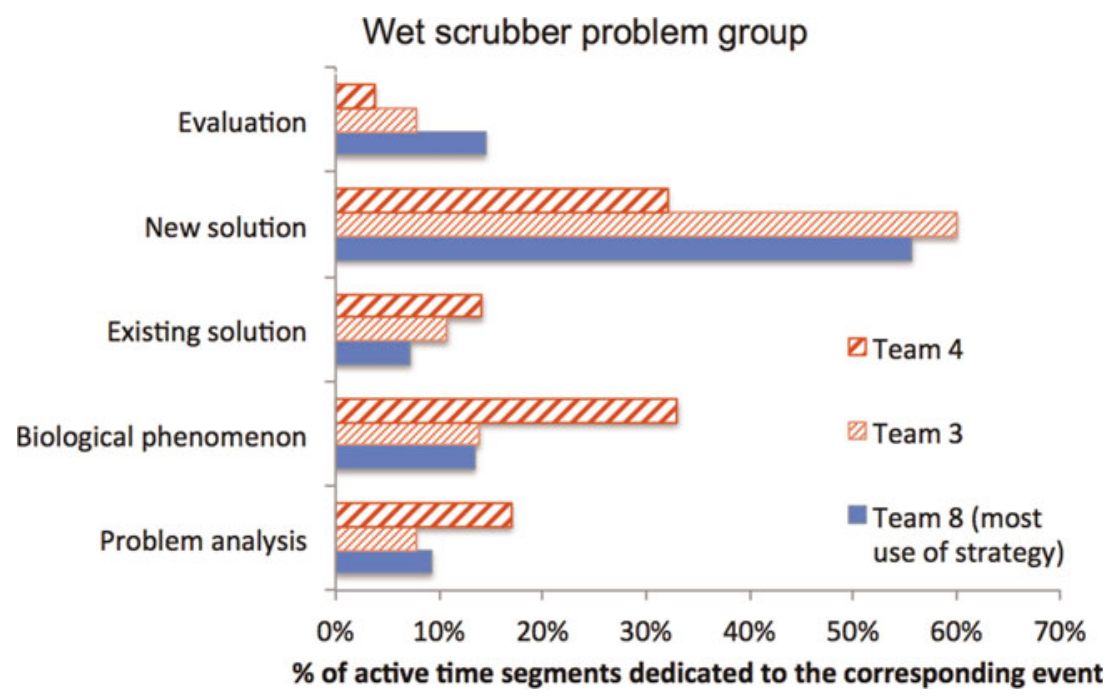

Fig. 9. Frequency of design activity modes by the three teams in wet scrubber problem group.

each problem group, revealing that the proportion of each design activity mode was similar between the three groups, and none of the differences were statistically significant.

\subsection{Comparing more strategic and less strategic teams}

Because the comparisons within problem groups were performed between individual teams, we could not calculate statistical significance between teams that developed more versus fewer new solutions using strategy-level comparisons. We therefore split the nine teams (across problem groups) according to the percentage of new solution generation segments that involve strategy-level comparisons. This percentage was calculated by dividing the number of design segments that involved both strategy-level comparisons and new solution generation by the number of total segments spent on new solution generation. Table 5 ranks and divides the teams into two groups based on how frequently they relied on the underlying strategy to develop new solutions. Team 8, with the median value of $14.6 \%$, was assigned to the more strategic group because its measure was closer to the smallest measure in this group than the largest measure in the less strategic group. To statistically confirm differences in design activity modes observed between the more strategic and less strategic groups, the Mann-Whitney $U$ test was used because the data was nonparametric. Although the less strategic group also developed analogous solutions, it did so very rarely compared with the more strategic group.

Figure 12 shows differences in the frequency of each design activity mode between the more strategic and less strategic groups. We observed that the more strategic group spent significantly less time recalling existing solutions than did the less strategic group $[U(9)=20.0, Z=2.45, p=$ 0.016]. The more strategic group also tended to spend more

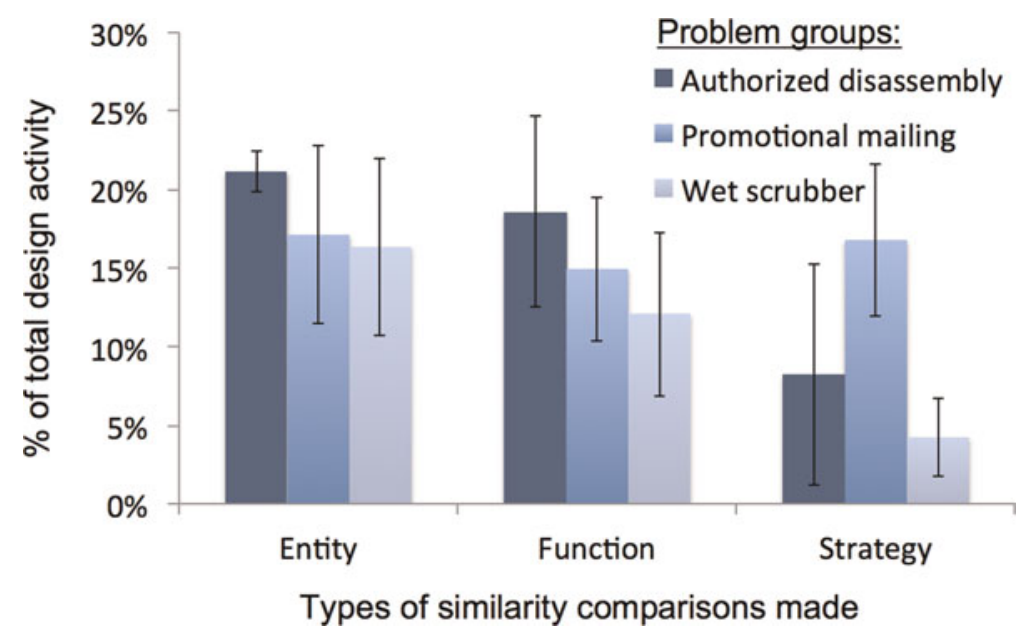

Fig. 10. Frequency of similarity comparisons by problem groups. Error bars represent 1 SE. 


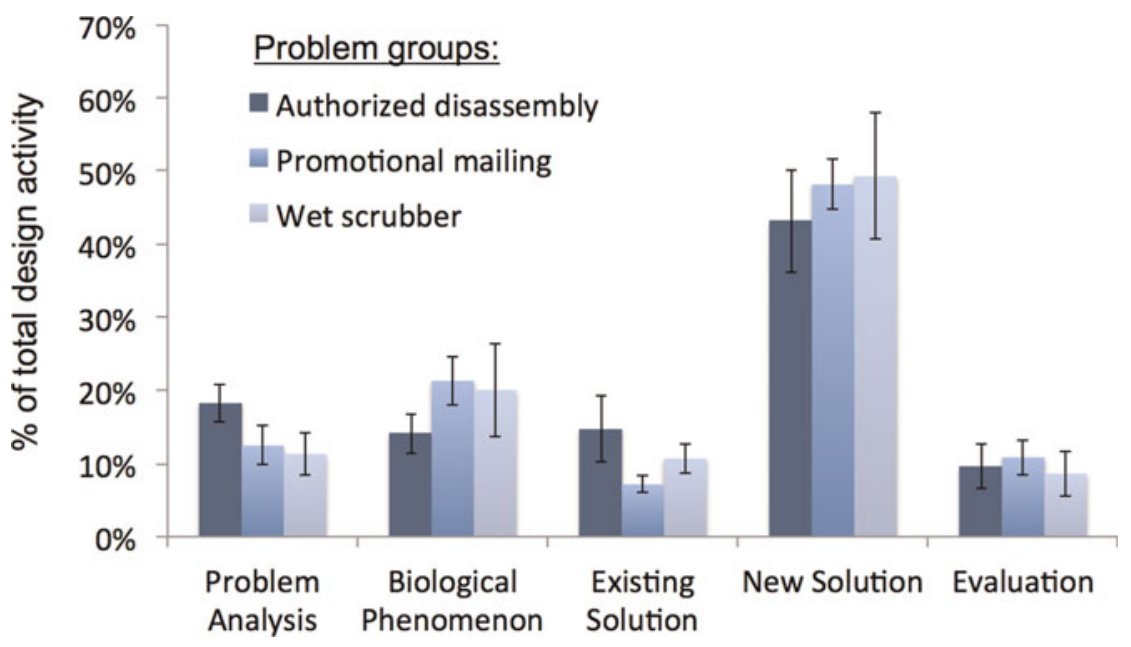

Fig. 11. Frequency of design activity modes by problem groups. Error bars represent 1 SE.

Table 5. Ranking and division of design teams by the percentage of new solution generation segments involving strategy-level comparisons

\begin{tabular}{clcl}
\hline \hline $\begin{array}{c}\text { Design } \\
\text { Team } \\
\text { No. }\end{array}$ & \multicolumn{1}{c}{ Design Problem } & $\begin{array}{c}\text { New Solution } \\
\text { Gen. Seg. }\end{array}$ & Assigned Group \\
\hline 1 & Promotional mailing & 38.7 & More strategic \\
2 & Authorized disassembly & 32.3 & More strategic \\
5 & Promotional mailing & 22.6 & More strategic \\
9 & Promotional mailing & 16.6 & More strategic \\
8 & Wet scrubber & 14.6 & More strategic \\
7 & Authorized disassembly & 7.6 & Less strategic \\
4 & Wet scrubber & 5.4 & Less strategic \\
3 & Wet scrubber & 2.1 & Less strategic \\
6 & Authorized disassembly & 0.0 & Less strategic \\
\hline \hline
\end{tabular}

time on evaluation than did the less strategic group. This difference was on the borderline of statistical significance $[U(9)$ $=18.0, Z=1.96, p=0.063]$.

Some of this group effect can be attributed to problem effect (i.e., the more strategic group contains all three teams that solved the promotional mailing problem). This likely problem effect will be discussed in the following section. However, Figure 11 shows no apparent differences in the time spent on evaluation among problem groups; therefore, this particular measure appears independent of the problem effect.

\section{DISCUSSION}

The results section identified problem-specific and problemgeneral tendencies that could be related to developing analogous solutions. In all three problem groups, the teams that

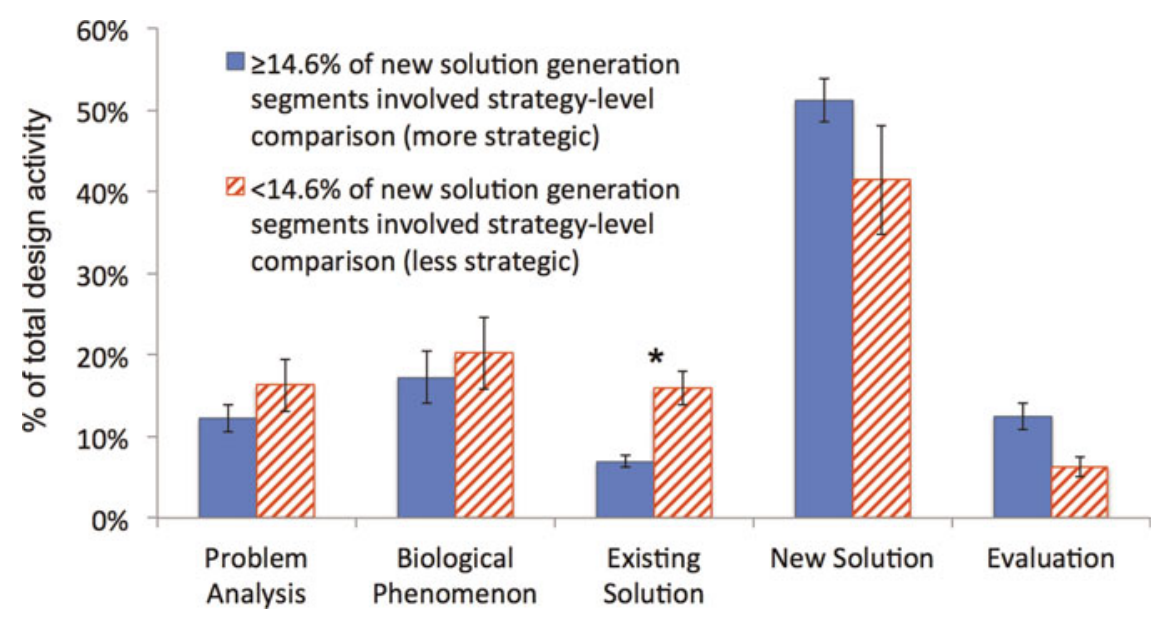

Fig. 12. Frequency of design activity modes between the more and less strategic groups (as defined in Table $5, * p<0.05$ ). Error bars represent $1 \mathrm{SE}$. 
spent the most time making strategy-level comparisons also tended to spend more time evaluating their solutions and less time recalling existing solutions.

The relationship between making strategy-level comparisons and entity- or function-level comparisons differed between the problems. For the authorized disassembly and the wet scrubber problems, the teams that made strategy-level comparisons the most also had the highest function-level comparisons relative to entity-level comparisons. For the promotional mailing problem, the team that made the least amount of both entity- and function-level comparisons made strategy-level comparisons the most.

The current section discusses these findings supported with qualitative observations made from the protocols. The following sections discuss the effect of observed behaviors on developing analogous solutions.

\subsection{Tendency to evaluate solutions}

Figures 4-9 indicate that the teams that developed analogous solutions the most also evaluated their solutions the most. We propose the following explanation for this correlation.

More frequent evaluation of ideas could be associated with a greater tendency to engage in critical thinking. In education research, Dirks (1998) observes that "critical thinking is most clearly involved in, and incorporates, analogical reasoning." Magliano and Pillow (2002) also point out that "causal reasoning is an important part of critical thinking." In contrast, using analogies in design has been seen as a means to form wild, creative ideas (e.g., as in Synectics; Gordon, 1961), and we are taught to suspend critical judgment during concept generation.

In biomimetic design, critical thinking could help designers look not only for similarities between design problems and biological phenomena but also for differences. Designers must determine which relations should be transferred to the solution domain, while also identifying which relations and objects are irrelevant. Therefore, we believe that critical thinking is an essential trait for analogical reasoning. Looking for associations without qualification can lead to incorrect analogical transfer and fixation on low-level characteristics of biological phenomena.

Critical thinking is also essential for designers to detect their own fixation or nonanalogous associations. One particular participant who exhibited this behavior belonged to Team 2, which solved the authorized disassembly problem and frequently used the underlying strategy to generate concepts. The participant repeatedly asked questions to himself and other team members about whether they were fixating on specific aspects of the design problem, as well as how they could apply the biological phenomenon in new ways. These types of questions likely contributed to the team's success in generating new solutions based on the corresponding strategy. Winkelmann and Hacker (2006) noted that design performance is increased through the use of interrogative questions, which stimulate reconsideration of the problem. Chrysikou and Weisberg (2005) and Linsey et al. (2010) developed defixation materials that specify solutions to avoid and list potential solution modes, respectively. We propose that these techniques should also guide designers to critique their own design behaviors, not just the solutions that they generate.

\subsection{Tendency to recall existing solutions}

Figures 4-9 indicate that teams developing analogous solutions the least also spent the most time recalling existing solutions.

For the authorized disassembly problem, we observed that participants frequently associated different characteristics of the enzyme-substrate interaction to their prior domain knowledge in engineering. For example, participants from Teams 6 and 7 developed solutions that use product-specific fasteners or part interfaces after uttering phrases such as "specific shape" and "binding to substrate." Examples of existing solutions referenced from the automotive and electronic industries included components that can only be accessed with specialized tools made by the OEM or Apple using unique interfaces for peripheral connections. Developing solutions based on familiar domain knowledge is consistent with Purcell and Gero's (1996) finding that mechanical engineers tend to fixate on familiar principles to solve design problems. Wiley (1998) reported that expert knowledge could actually promote fixation in creative problem solving, especially when the desired solution does not reside in the problem solver's existing knowledge.

This tendency to recall existing solutions could have prevented participants from considering the underlying strategy suggested in the biological phenomenon. Specifically, participants fixated on using specialized fasteners or tools, but they did not consider how environmental changes could facilitate disassembly. The following section presents this argument in more detail.

\subsection{Biological phenomena and levels of comparisons}

The majority of existing solutions recalled from prior knowledge did not use the strategy as a whole. These solutions seem to have been inspired from mostly specific superficial characteristics of the biological phenomenon without incorporating the overall analogy. Table 6 shows similarities between potential solutions for the design problem and the biological phenomenon at the superficial, functional, and strategy levels. We believe that for the authorized disassembly problem, the participants were more likely to develop solutions based on the entity- or functional-level characteristics of the biological phenomenon because they could be easily associated with existing solutions. After developing readily available solutions, the participants may have fixated on those solutions and overlooked considering the overall analogy.

The same reasoning could be used to explain why the promotional mailing problem group was better at incorporating the overall analogy in its solutions. Table 7 shows that for 
Table 6. Examples of analogous elements between the enzyme-substrate interaction and potential solutions for the authorized disassembly problem at three levels of comparison

\begin{tabular}{lccc}
\hline \hline $\begin{array}{c}\text { Comparison } \\
\text { Level }\end{array}$ & $\begin{array}{c}\text { Enzyme-Substrate } \\
\text { Interaction }\end{array}$ & $\begin{array}{c}\text { Authorized } \\
\text { Disassembly }\end{array}$ & Similarity \\
\hline Strategy & $\begin{array}{c}\text { Bind based on } \\
\text { specific substrate; } \\
\text { temperature } \\
\text { changes the shape } \\
\text { of enzyme to } \\
\text { release }\end{array}$ & $\begin{array}{c}\text { Assemble based on } \\
\text { specific part } \\
\text { interface; } \\
\text { temperature } \\
\text { changes the shape } \\
\text { of part interface to } \\
\text { disassemble }\end{array}$ & Yes \\
Functional & $\begin{array}{c}\text { Binding of enzyme } \\
\text { to substrate } \\
\text { Attaching of one part } \\
\text { to another }\end{array}$ & Yes \\
Superficial & $\begin{array}{c}\text { Specific shape of } \\
\text { substrate }\end{array}$ & interface & Yes \\
& & & \\
\hline \hline
\end{tabular}

Note: All three levels of comparison feature some degree of similarity.

the promotional mailing problem, the analogous elements at the superficial and functional levels have little similarity. The participants therefore could not associate the lower level characteristics of the ant foraging phenomenon to existing solutions that could be used to solve the problem. This lack of available association may have led the participants to identify the underlying strategy.

Figure 13 shows a coded portion of design segments for Team 1, who solved the promotional mailing problem while making the fewest entity- and function-level comparisons and the most strategy-level comparisons. Most of this team's new solutions were generated based on strategy-level comparisons. No existing solutions were recalled in this sequence.

Figure 14 shows a contrasting sequence from Team 7 , which worked on the authorized disassembly problem. The team continuously made entity- and functional-level comparisons, simultaneously or subsequently recalling existing solutions.

In summary, the tendency to recall existing solutions could prevent novice designers from identifying underlying strategies in biological phenomena. Based on our qualitative observations, this tendency is more likely when readily available

Table 7. Examples of analogous elements between the ant foraging phenomenon and potential solutions for the promotional mailing problem at three levels of comparison

\begin{tabular}{|c|c|c|c|}
\hline $\begin{array}{c}\text { Comparison } \\
\text { Level }\end{array}$ & Ant Foraging & Promotional Mailing & Similarity \\
\hline Strategy & $\begin{array}{l}\text { Target food source } \\
\text { based on feedback } \\
\text { obtained from } \\
\text { random travel }\end{array}$ & $\begin{array}{l}\text { Target sign-ups based } \\
\text { on feedback } \\
\text { obtained from } \\
\text { random mailing }\end{array}$ & Yes \\
\hline Functional & $\begin{array}{l}\text { Traveling to food } \\
\text { source }\end{array}$ & Sending out mail & No \\
\hline Superficial & Food source & Sign-ups & No \\
\hline
\end{tabular}

Note: Only the strategy level of comparison features a relational similarity.

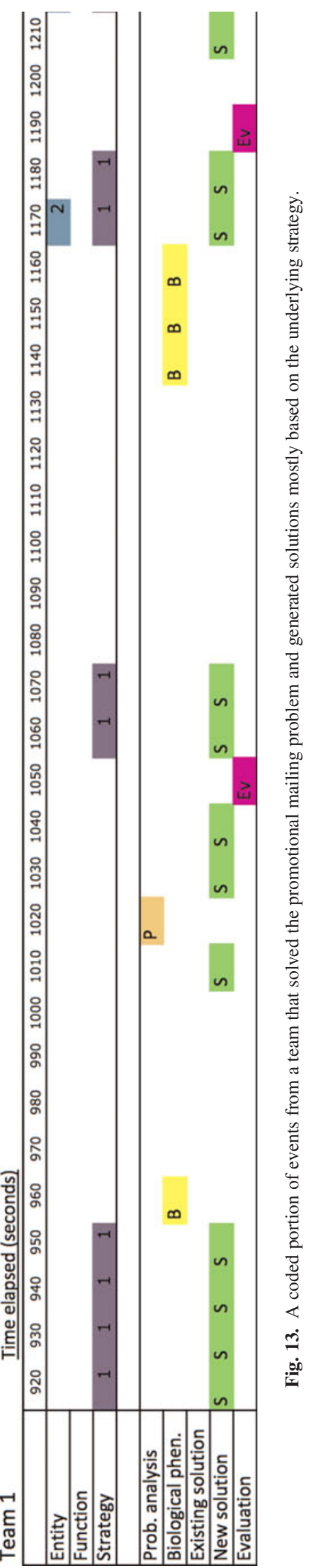


associations exist at the lower levels of comparison between the biological phenomena and prior knowledge.

\subsection{Other contributions to nonanalogous solution generation}

Figures 4, 5, 8, and 9 suggest that the teams that generated fewer solutions based on underlying strategies tended to rely more on entity-level comparisons relative to functionlevel comparisons. The tendency to rely on entity-level comparisons suggests fixation on specific entities of biological phenomena. Previous research in biomimetic design reports that such fixation can prevent detecting and applying the overall analogy (Mak \& Shu, 2008; Helms et al., 2009; Cheong et al., 2010; Cheong \& Shu, 2013b).

\subsubsection{Fixation on biological entities}

Fixation on superficial characteristics of biological phenomena was often observed. Figure 15 shows design segments of Team 4, which worked on the wet scrubber problem and had one participant who exclusively found associations at the entity level. This participant persistently tried to apply superficial characteristics of a penguin's feet (e.g., shape, texture, and color) to develop new types of mechanical scrubbers). The only instances of strategy-level comparisons were made by another team member; however, these instances did not stop the first participant from fixating on superficial characteristics.

One could conclude that the participant who fixated on superficial characteristics was consistently using associative thinking, a behavior often considered to be important for creative design. However, biomimetic design requires a specific type of associative thinking: analogical reasoning. The current study suggests that associative thinking that only focuses on either a specific form or a function can prevent analogical reasoning in biomimetic design; it may benefit designers to suppress associations with low-level characteristics of biological phenomena and focus on finding similarities in the underlying strategy. Cheong and Shu (2013b) investigated the effects of abstracting nouns in descriptions of biological phenomena to reduce such associations.

\subsubsection{Mapping additional entities between design problems and biological phenomena}

In some cases, participants developed solutions based on the underlying strategy, but they tried to look for additional entities that could be mapped between design problems and biological phenomena. Their conversations suggested that they felt that the initial analogy detected was incomplete.

Teams 5 and 9 of the promotional mailing problem group started to make irrelevant associations (e.g., identifying the optimal path to deliver mail or comparing a CEO to a queen ant) after they had detected the underlying strategy. Identifying the optimal path was not the problem goal specified, and entities such as queen ants were not present in the descrip-

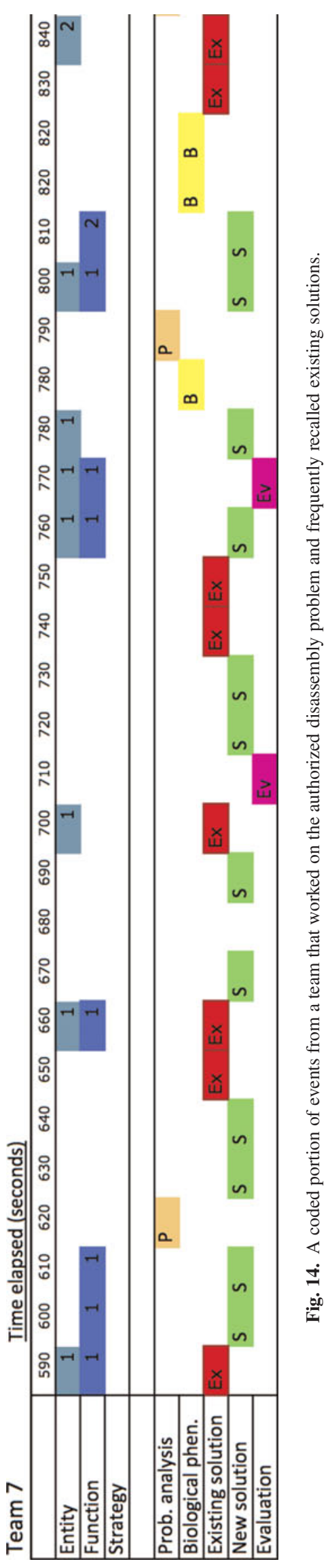




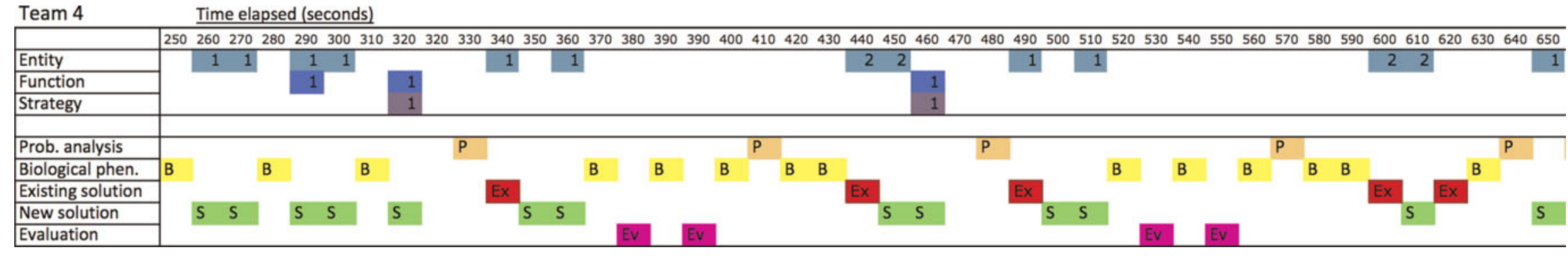

Fig. 15. A coded portion of events from a team that solved the wet scrubber problem and fixated on biological entities.

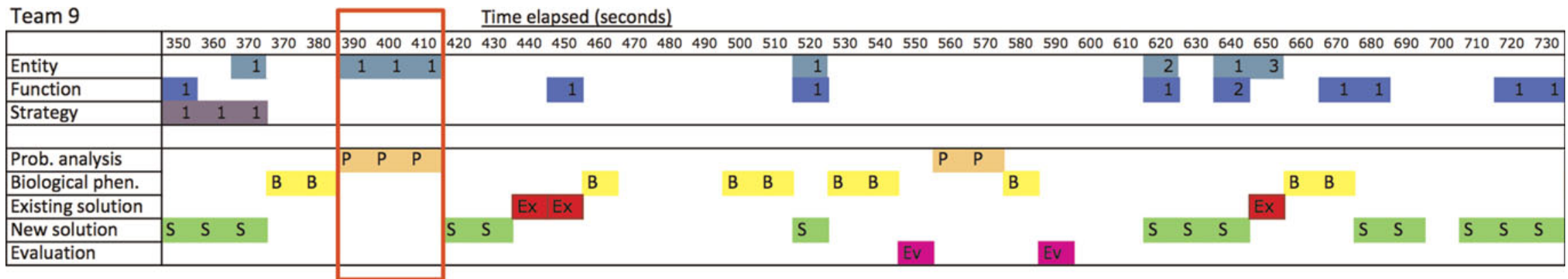

Fig. 16. Coded events from team that worked on the promotional mailing problem; the box contains instances of comparison of entities between the problem and the biological phenomenon. 


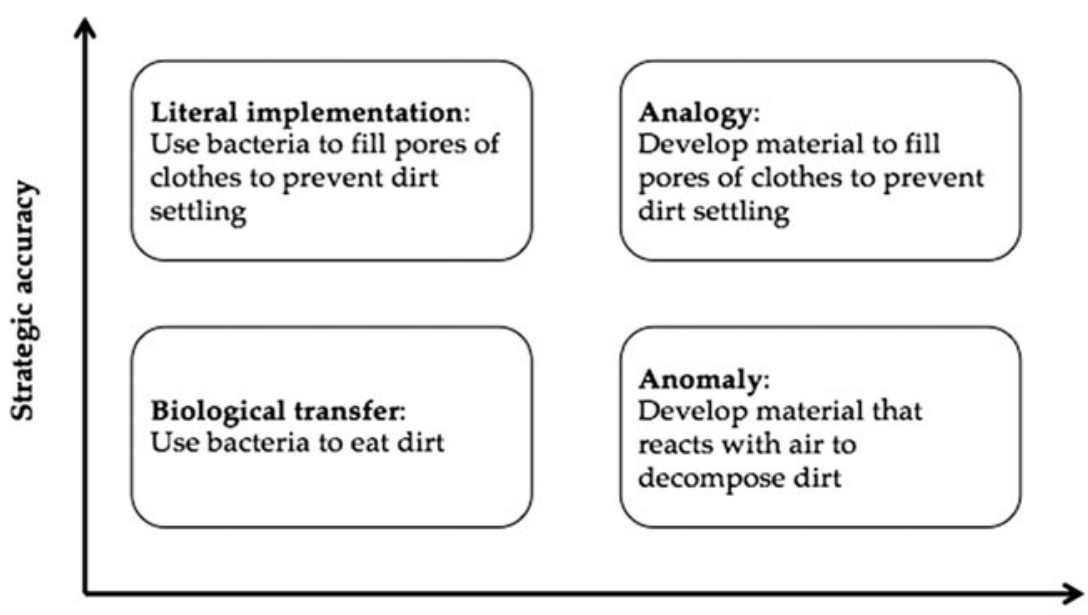

Abstraction of biological entities

Fig. 17. Types of correspondence between biological phenomena and developed concepts. Adapted from Mak and Shu (2004).

tions provided. Figure 16 shows a sequence of design segments from Team 9 that included this behavior. After the comparisons between the biological phenomenon and the design problem occurred, the subsequent solution generation was based on comparisons at either the entity or the functional level and not at the strategy level.

Team 3, which worked on the wet scrubber problem, also showed a similar tendency. After agreeing to use countercurrent flow exchange (underlying strategy), the team tried to elaborate its solution with irrelevant associations based on the penguin's circulatory system (e.g., using veinlike channels and considering the distance between the penguin's heart and feet).

The participants were likely looking for more one-to-one mappings from the analog source (Holyoak \& Thagard, 1989; Krawczyk et al., 2005), instead of generating multiple solutions based on the analogy. This particular tendency could have occurred because the participants were asked to use a specific biological phenomenon as the source of analogy. In practice, designers may not make irrelevant associations once they detect an analogy and develop a corresponding solution. However, the tendency to focus on entities of a biological phenomenon, which likely propagated the tendency to look for additional one-to-one mappings, may well hinder designers from detecting the underlying strategy.

\subsection{Insights from nonaudiorecorded team}

As mentioned in Section 3, a group of four students were uncomfortable being audiorecorded, but they consented to have insights that arose from their participation in the design exercise qualitatively reported. These students remarkably demonstrated a concept-generation strategy based on the different types of correspondence that the observers were instructed to determine and evaluate in the active team.

Figure 17 shows the types of correspondence described immediately preceding the experiment. Definitions of these types of correspondence are given following the example of developing concepts that result in "clean clothes," by using the description of a biological phenomenon by Purves et al. (2001): "Barriers and local agents defend the body: skin is a primary innate defense against invasion. The bacteria and fungi that normally live and reproduce in great numbers on our body surfaces without causing disease are referred to as normal flora. These natural occupants of our bodies compete with pathogens for space and nutrients, so normal flora are a form of innate defense."

The vertical axis of Figure 17 corresponds to whether the developed solution accurately shares the underlying strategy of the biological phenomenon provided. The horizontal axis of Figure 17 corresponds to whether the biological entities (e.g., bacteria/fungi) were abstracted or used literally in the developed solution.

1. A literal implementation involves using biological entities (e.g., bacteria) directly to solve the engineering problem (e.g., by filling clothing pores to prevent dirt from settling). Here, the biological entities are not abstracted, but rather, used directly, with the same strategy between source and problem domains.

2. A biological transfer involves transferring the biological entities (e.g., bacteria) into the solution domain but without applying the strategy presented in the biological domain. For example, bacteria are used to provide the solution of clean clothes by eating dirt.

3. An anomalous solution involves neither the entities nor the strategy from the biological phenomenon. Some anomalous concepts are due to lack of understanding of, or fixation on a few words in, the text description while disregarding the underlying strategy.

4. The intended analogous solution applies the strategy from the biological phenomenon to the concept without transferring the biological entities (e.g., bacteria) into the solution. 
Because the observation of this group was done in person at the time of the activity, it was much easier to follow the work of single team members. One particular member of this group worked on the authorized disassembly problem, which was problematic for two thirds of the audiorecorded teams assigned this problem. He also had initial difficulty in developing an analogous solution but coped by starting with a literal implementation and working toward the analogous solution by abstracting the biological entities. Specifically, he began by describing $\mathrm{pH}$ - or temperature-sensitive enzymes used to hold together parts that require disassembly. He then abstracted the geometric binding between the enzyme and the substrate to geometric features of the parts to be disassembled while retaining the qualities of $\mathrm{pH}$ and temperature sensitivity. Thus, he spontaneously demonstrated a design process that may help novice designers who find it difficult to develop analogous solutions given a biological phenomenon.

\section{CONCLUSION}

The current research conducted a verbal protocol analysis to examine design problem solving with biological analogies. The results suggest that recalling existing solutions from prior knowledge prevented novice designers from detecting the overall analogy. Qualitative observations suggest that this effect could be attributed to associations made at low levels of comparison (e.g., superficial and functional), and the availability of these associations depend on the design problem and corresponding biological phenomenon. The tendency to self-evaluate has been shown to help novice designers reduce associations at the lower levels of comparison and increase associations at the higher, strategic level needed for analogical reasoning. In general, we argue that analogical reasoning in biomimetic design is not a simple associative task but rather a complex process that requires critical thinking to appropriately identify similarities and differences between two concepts.

Logistical challenges introduced some limitations to the experimental setup of the current research. An experiment with a larger sample size would have provided greater statistical power and strengthened the confidence in the observations made. More problem-solving time given to participants would have also increased the quantity of protocol data collected from each team, which could have enabled intrasession data analysis across time. Potential confounding effects (e.g., participants observing other participants solve different design problems or overhearing other participants solving the same problems) should be mitigated in future studies.

Other ideas for future studies include presenting multiple sources of biological analogies or letting designers choose their own biological analogies. In either case, it would be interesting to examine whether or not designers would still focus on making low-level associations or rely on existing solutions to make connections between biological phenomena and design solutions. Representation modes other than text descriptions could also influence the types of associations that designers make, as reported by Sarkar and Chakrabarti (2008) and Helms et al. (2010). Expert designers and longer design sessions would complement the current work. Critical thinking skills of expert designers could help them detect analogies; however, their extensive knowledge base may also lead to more nonanalogous associations.

Despite these outstanding challenges, we believe that the current research elucidated some aspects of the complex cognition tasks involved in analogical reasoning for biomimetic design. These research findings can contribute toward developing methods that facilitate and support biomimetic design.

\section{ACKNOWLEDGMENTS}

We thank the Natural Sciences and Engineering Research Council of Canada for financial support. We also thank the students of MIE440 at the University of Toronto for participating in this study.

\section{REFERENCES}

Bilda, Z., \& Gero, J.S. (2008). Idea development can occur using imagery only. In Design Computing and Cognition '08 (Gero, J.S., Ed.), pp. 303-320. New York: Springer.

Chakrabarti, A., Sarkar, P., Leelavathamma, B., \& Nataraju, B.S. (2005). A functional representation for aiding biomimetic and artificial inspiration of new ideas. Artificial Intelligence for Engineering Design, Analysis and Manufacturing 19(2), 113-132.

Cheong, H.M., Chiu, I., \& Shu, L.H. (2010). Extraction and transfer of biological analogies for creative concept generation. Proc. ASME 2010 Int Design Engineering Technical Conf. Computers and Information in Engineering Conf., Paper No. IDETC2010/DTM-29006, Montreal, August 15-18.

Cheong, H.M., \& Shu, L.H. (2009). Effective analogical transfer using biological descriptions retrieved with functional and biologically meaningful keywords. Proc. ASME 2009 Int. Design Engineering Technical Conf. Computers and Information in Engineering Conf., Paper No. IDETC2009/DTM-86680, San Diego, CA, August 30-September 2.

Cheong, H.M, \& Shu, L.H. (2013a). Using templates and mapping strategies to support analogical transfer in biomimetic design. Design Studies 34(6), 706-728.

Cheong, H.M., \& Shu, L.H. (2013b). Reducing cognitive bias in biomimetic design by abstracting nouns. CIRP Annals 62, 111-114.

Chiu, I., \& Shu, L.H. (2010). Potential limitations of verbal protocols in design experiments. Proc. ASME 2010 Int. Design Engineering Technical Conf. Computers and Information in Engineering Conf., Paper No. IDETC2010/DTM-28675, Montreal, August 15-18.

Chrysikou, E.G., \& Weisberg, R.W. (2005). Following the wrong footsteps: fixation effects of pictorial examples in a design problem-solving task. Journal of Experimental Psychology: Learning, Memory, and Cognition 31(5), 1134-1148.

Cross, N., Christians, H., \& Dorst, K. (1996). Introduction: the Delft Protocols Workshop. In Analysing Design Activity (Cross, N., Christians, H., \& Dorst, K., Eds.), pp. 1-14. West Sussex: Wiley.

Davidson, M., Bligh, D., Maloney, N., McKnight, C., Young, W., Shu, L., Potvin, M.-J., \& Warkentin, A. (2009). Biomimetic design of a multilayered dust protection system for optical instruments operating in the lunar environment. Proc. 6th Int. Conf. Innovation \& Practices in Eng. Design \& Eng. Education, Hamilton, ON, July 27-29.

Dinar, M., Maclellan, C., Danielescu, A., Shah, J.J., \& Langley, P. (2012). Beyond function-behavior-structure. Proc. 5th Int. Conf. Design Computing and Cognition DCC'12, College Station, TX, June 7-9.

Dirks, A.L. (1998). Critical thinking and analogical thinking: a model. Accessed at http://webhost.bridgew.edu/adirks/ald/papers/analog.htm on September 11, 2012.

Dong, A. (2005). The latent semantic approach to studying design team communication. Design Studies 26(5), 445-461. 
Dong, A. (2006). Concept formation as knowledge accumulation: a computational linguistics study. Artificial Intelligence for Engineering Design, Analysis and Manufacturing 20(1), 35-53.

Dong, A. (2007). The enactment of design through language. Design Studies 28(1), 5-21.

Ericsson, K.A., \& Simon, H.A. (1993). Protocol Analysis: Verbal Reports as Data, rev. ed. Cambridge, MA: MIT Press.

Fleiss, J.L. (1981). Statistical Methods for Rates and Proportions, 2nd ed. New York: Wiley.

Gentner, D. (1983). Structure-mapping: a theoretical framework for analogy. Cognitive Science 7(2), 155-170.

Gentner, D. (2003). Analogical reasoning, psychology of. In Encyclopedia of Cognitive Science (Nadel, L., Ed.), Vol. 1, pp. 106-112. London: Nature Publishing Group.

Gero, J.S. (1990). Design prototypes: a knowledge representation schema for design. AI Magazine 11(4), 26-36.

Gero, J.S. (2010a). Generalizing design cognition. Proc. 8th Design Thinking Research Symp. (DTRS8), Sydney, October 19-20.

Gero, J.S. (2010b). Fixation and commitment while designing and its measurement. Journal of Creative Behavior 45(2), 108-115.

Gero, J.S., \& McNeill, T. (1998). An approach to the analysis of design protocols. Design Studies 19(1), 21-61.

Goel, A.K. (1997). Design, analogy, and creativity. IEEE Expert 123, 62-70.

Goel, A.K., Vattam, S., Wiltgen, B., \& Helms, M. (2011). Cognitive, collaborative, conceptual and creative-four characteristics of the next generation of knowledge-based CAD systems: a study in biologically inspired design. Computer-Aided Design 44(10), 879-900.

Goldschmidt, G. (1990). Linkography: assessing design productivity. In $C y$ bernetics and systems '90, Proc. 10th European Meeting on Cybernetics and Systems Research (Trappl, R., Ed.), pp. 291-298. Singapore: World Scientific.

Goldschmidt, G. (1995). The designer as a team of one. Design Studies 16(2), 189-209.

Gordon, W.J.J. (1961). Synectics: The Development of Creative Capacity. New York: Harper and Brothers.

Helms, M., Vattam, S.S., \& Goel, A.K. (2009). Biologically inspired design: process and products. Design Studies 30(5), 606-622.

Helms, M., Vattam, S.S., \& Goel, A.K. (2010). The effect of functional modeling on understanding complex biological systems. Proc. ASME 2010 Int. Design Engineering Technical Conf. Computers and Information in Engineering Conf., Paper No. IDETC2010/DTM-28939, Montreal, August 15-18.

Holyoak, K.J., \& Thagard, P.R. (1989). Analogical mapping by constraint satisfaction. Cognitive Science 13(3), 295-355.

Holyoak, K.J., \& Thagard, P.R. (1995). Mental Leaps: Analogy in Creative Thought. Cambridge, MA: MIT Press.

Jin, Y., \& Benami, O. (2010). Creative patterns and stimulation in conceptual design. Artificial Intelligence for Engineering Design, Analysis and Manufacturing 24(2), 191-209.

Kan, J.W.T., Bilda, Z., \& Gero, J.S. (2007). Comparing entropy measures of idea links in design protocols: linkography entropy measurement and analysis of differently conditioned design sessions. Artificial Intelligence for Engineering Design, Analysis and Manufacturing 21(4), 367-377.

Krawczyk, D.C., Holyoak, K.J., \& Hummel, J.E. (2005). The one-to-one constraint in analogical mapping and inference. Cognitive Science 29(5), 797-806.

Kvan, T., \& Gao, S. (2006). A comparative study of problem framing in multiple settings. In Design Computing and Cognition '06 (Gero, J.S., Ed.), pp. 245-263. New York: Springer.

Landis, J.R., \& Koch, G.G. (1977). The measurement of observer agreement for categorical data. Biometrics 33(1), 159-174.

Linsey, J.S., Tseng, I., Fu, K., Cagan, J., Wood, K.L., \& Schunn, C. (2010). A study of design fixation, its mitigation and perception in engineering design faculty. Journal of Mechanical Design 132(4), 041003.

Linsey, J.S., Wood, K.L., \& Markman, A.B. (2008). Modality and representation in analogy. Artificial Intelligence for Engineering Design, Analysis and Manufacturing 22(2), 85-100.

Linstone, H.A., \& Turoff, M. (1975). The Delphi Method: Techniques and Applications. Reading, MA: Addison-Wesley.

Magliano, J.P., \& Pillow, B.H. (2002). Learning: causal reasoning. In Encyclopedia of Education (Guthrie, J.W., Ed.), pp. 1425-1427. New York: Macmillan.

Mak, T.W., \& Shu, L.H. (2004). Abstraction of biological analogies for design. CIRP Annals 53(1), 117-120.
Mak, T.W., \& Shu, L.H. (2008). Using descriptions of biological phenomena for idea generation. Research in Engineering Design 19(1), 21-28.

Markman, A.B., \& Gentner, D. (1993). Structural alignment during similarity comparisons. Cognitive Psychology 25(4), 431-467.

Merriam, S. (2009). Qualitative Research: A Guide to Design and Implementation, 3rd ed. San Francisco, CA: Jossey-Bass.

Miles, M.B., \& Huberman, A.M. (1994). Qualitative Data Analysis: An Expanded Sourcebook, 2nd ed. Thousand Oaks, CA: Sage.

Nagel, J.K.S., Nagel, R.L., Stone, R.B., \& McAdams, D.A. (2010). Function based, biologically inspired concept generation. Artificial Intelligence for Engineering Design, Analysis and Manufacturing 24(4), 521-535.

Nagel, J.K.S., Stone, R.B., \& McAdams, D.A. (2010). An engineeringto-biology thesaurus for engineering design. Proc. ASME 2010 Int. Design Engineering Technical Conf. Computers and Information in Engineering Conf., Paper No. IDETC2010/DTM-28233, Montreal, August $15-18$.

Purcell, A.T., \& Gero, J.S. (1996). Design and other types of fixation. Design Studies 17(4), 363-383.

Purves, W.K., Sadava, D., Orians, G.H., \& Heller, H.C. (2001). Life, the Science of Biology, 6th ed. Sunderland, MA: Sinauer Associates.

Saitou, K., Shalaby, M., \& Shu, L.H. (2007). Bioanalogous mechanical joints for authorized disassembly. CIRP Annals 56(1), 33-36.

Sarkar, P., \& Chakrabarti, A. (2008). The effect of representation of triggers on design outcomes. Artificial Intelligence for Engineering Design, Analysis and Manufacturing 22(2), 101-116.

Sartori, J., Pal, U., \& Chakrabarti, A. (2010). A methodology for supporting "transfer" in biomimetic design. Artificial Intelligence for Engineering Design, Analysis and Manufacturing 24(4), 483-505.

Schön, D.A. (1983). The Reflective Practitioner: How Professionals Think in Action. New York: Basic Books.

Shu, L.H. (2010). A natural-language approach to biomimetic design. Artificial Intelligence for Engineering Design, Analysis and Manufacturing 24(4), 507-519.

Shu, L.H., Lenau, T.A., Hansen, H.N., \& Alting, L. (2003). Biomimetics applied to centering in microassembly. CIRP Annals 52(1), 101-104.

Shu, L.H., Ueda, K., Chiu, I., \& Cheong, H. (2011). Biologically inspired design. CIRP Annals 60(2), 673-693.

van Someren, M.W., Barnard, Y.F. \& Sandberg, J.A.C. (1994). The Think Aloud Method: A Practical Guide to Modelling Cognitive Processes. London: Academic Press.

Vattam, S.S., Helms, M.E., \& Goel, A.K. (2010). A content account of creative analogies in biologically inspired design. Artificial Intelligence for Engineering Design, Analysis and Manufacturing 24(4), 467-481.

Vattam, S.S., Wiltgen, B., Helms, M., Goel, A., \& Yen, J. (2010). DANE: fostering creativity in and through biologically inspired design. Proc. 1st Int. Conf. Design Creativity, Kobe, Japan, 127-132.

Ward, T. (1998). Mental leaps versus mental hops. In Advances in Analogy Research: Integration of Theory and Data from the Cognitive, Computational, and Neural Sciences (Holyoak, K.J., Gentner, D., \& Kokinov, B.N., Eds.), pp. 221-230. Sofia, Bulgaria: New Bulgarian University Press.

Wiley, J. (1998). Expertise as mental set: the effects of domain knowledge in creative problem solving. Memory \& Cognition 26(4), 716-730.

Winkelmann, C., \& Hacker, W. (2006). The improvement of design solutions by means of a question-answering technique. In Design Computing and Cognition '06 (Gero, J.S., Ed.), pp. 603-618. New York: Springer.

Hyunmin Cheong recently completed his $\mathrm{PhD}$ at the University of Toronto. He obtained his MS and BS in mechanical and industrial engineering at the University of Toronto. His research interests include applying natural-language processing techniques to support biomimetic design and understanding designers, analogical reasoning, and creativity.

Gregory M. Hallihan obtained his MS in mechanical and industrial engineering at the University of Toronto and his BA in psychology at the University of Calgary. His research focuses on applying literature from psychology to better understand design cognition. 
L.H. Shu is an Associate Professor in the Department of Mechanical and Industrial Engineering at the University of Toronto, where she held the Wallace G. Chalmers Chair of Engineering Design. She obtained her PhD and MS from MIT and her BS from the University of Nevada, all in mechanical engineering. She is a fellow of the International Academy for
Production Engineering (CIRP) and received the F.W. Taylor Medal Award in 2004. Her research interests include creativity in conceptual design, systematic identification and application of biological analogies in biomimetic (biologically inspired) design, and identifying and overcoming obstacles to personal environmentally conscious behavior. 